\title{
Experimental verification of a Monte Carlo-based MLC simulation model for IMRT dose calculation
}

\author{
Neelam Tyagi, ${ }^{\text {a) }}$ Jean M. Moran, and Dale W. Litzenberg \\ The University of Michigan, Department of Radiation Oncology, Ann Arbor, Michigan 48109-0010 \\ Alex F. Bielajew \\ The University of Michigan, Department of Nuclear Engineering and Radiological Sciences, Ann Arbor, \\ Michigan 48109-2104 \\ Benedick A. Fraass and Indrin J. Chetty ${ }^{\text {b) }}$ \\ The University of Michigan, Department of Radiation Oncology, Ann Arbor, Michigan 48109-0010
}

(Received 6 June 2006; revised 25 October 2006; accepted for publication 5 December 2006; published 26 January 2007)

\begin{abstract}
Inter- and intra-leaf transmission and head scatter can play significant roles in intensity modulated radiation therapy (IMRT)-based treatment deliveries. In order to accurately calculate the dose in the IMRT planning process, it is therefore important that the detailed geometry of the multi-leaf collimator (MLC), in addition to other components in the accelerator treatment head, be accurately modeled. In this paper, we have used the Monte Carlo method (MC) to develop a comprehensive model of the Varian 120 leaf MLC and have compared it against measurements in homogeneous phantom geometries under different IMRT delivery circumstances. We have developed a geometry module within the DPM MC code to simulate the detailed MLC design and the collimating jaws. Tests consisting of leakage, leaf positioning and static MLC shapes were performed to verify the accuracy of transport within the MLC model. The calculations show agreement within $2 \%$ in the high dose region for both film and ion-chamber measurements for these static shapes. Clinical IMRT treatment plans for the breast [both segmental MLC (SMLC) and dynamic MLC (DMLC)], prostate (SMLC) and head and neck split fields (SMLC) were also calculated and compared with film measurements. Such a range of cases were chosen to investigate the accuracy of the model as a function of modulation in the beamlet pattern, beamlet width, and field size. The overall agreement is within $2 \% / 2 \mathrm{~mm}$ of the film data for all IMRT beams except the head and neck split field, which showed differences up to 5\% in the high dose regions. Various sources of uncertainties in these comparisons are discussed. (C) 2007 American Association of Physicists in Medicine.
\end{abstract}

[DOI: $10.1118 / 1.2428405]$

Key words: Monte Carlo, multi-leaf collimator, dose calculation, IMRT

\section{INTRODUCTION}

Dose calculations for MLC-based intensity modulated radiation therapy (IMRT) planning are quite challenging, primarily because dose distributions are sensitive to the detailed structure of the multileaf collimator (MLC). Detailed modeling of MLCs requires modeling inter and intra-leaf transmission, rounded leaf ends (on Varian MLCs), and consideration of the tongue and groove effect. ${ }^{1}$ The Monte Carlo (MC) method offers a distinct advantage compared with other dosimetric algorithms, ${ }^{2,3}$ especially in IMRT, because in addition to its improved accuracy in heterogeneous media, MC can also perform accurate simulation of the detailed treatment head geometry. ${ }^{4-6}$ After proper experimental verification, the MC method may be a useful tool for IMRT quality assurance (QA), particularly in heterogeneous phantoms or in the patient geometries where measurements for routine QA are impractical.

Various Monte Carlo codes ${ }^{4,7-9}$ have been developed to simulate the detailed geometries of different types of MLCs. The pioneer among them is the BEAM code where various component modules specific for MLC such as MLC, MLCQ and VARMLC ${ }^{4}$ (which differ in their ability to model the straight edge or rounded edge or model the 80 leaf MLC, respectively) have been written to accurately simulate conformal and intensity modulated beams. Liu et l. $^{7}$ used the MLCQ component module to model 120-leaf MLC and used it for segmental MLC (SMLC) and dynamic (DMLC) delivery. Kim et al. ${ }^{9}$ used the MCNP code to model the Millennium 120 leaf MLC. Recently Heath and Seuntjens ${ }^{8}$ have developed a detailed model of the 120 leaf MLC within the BEAM code (called DYNMLC) and have shown validation comparisons pertaining to static and dynamic IMRT beams.

Other investigators have used a source model approach to simulate IMRT beams: Pawlicki et al. ${ }^{10}$ use an intensity grid for each IMRT field from MLC leaf-sequence files. The leaf transmission is accounted for by adding a fraction of the monitor units for the closed beamlets in each leaf step to the intensity map. The method thus does not account for explicit transport through the MLC. Siebers et al. ${ }^{11}$ used a first order Compton scatter approximation for photon only transport in their MLC model. Electron transport and pair production is ignored as these effects form a very small fraction of total 


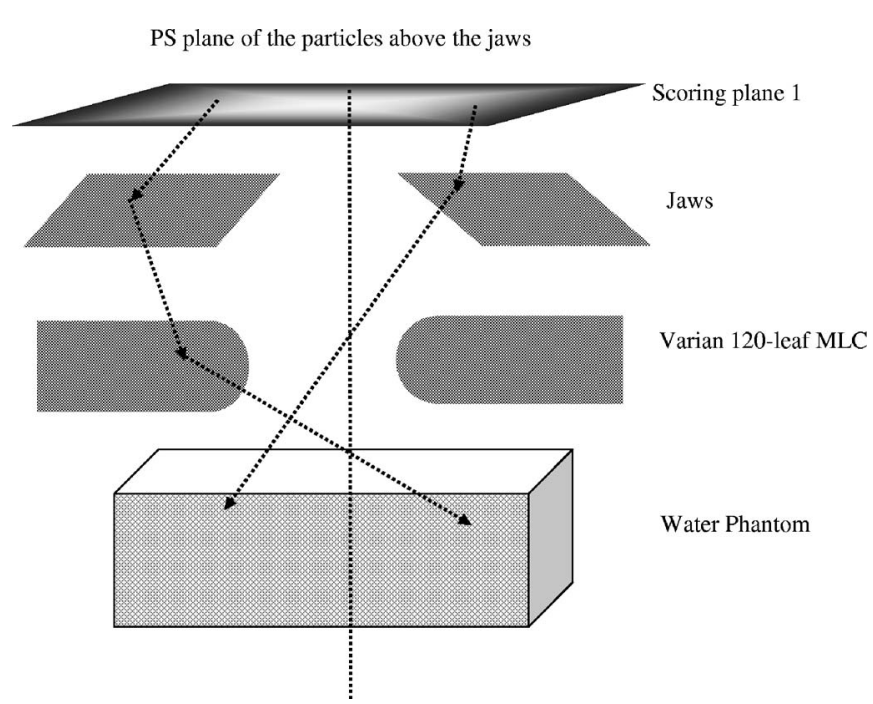

FIG. 1. Patient dependent structures modeled using DPM Monte Carlo code.

fluence. These effects, though, might be significant for electrons that are generated close to the bottom edge of the leaf which are likely to affect the dose in the buildup region. Aaronson et al. $^{12}$ modified a previous phase-space (PS) source model developed by Chetty et al. ${ }^{13}$ to simulate IMRT fields for a Novalis accelerator. They calculate an average path length through the leaves and modify the incident intensity with this average transmission.

In this study, we have developed a geometry module within the DPM MC code, ${ }^{14-16}$ in order to perform transport through the jaws and MLC for the purposes of IMRT dose calculation and verification. The DPM-based transport model of the Millennium MLC (120 leaf MLC, Varian Medical Systems, Palo Alto, CA) is compared against ion chamber and film measurements for SMLC and DMLC IMRT deliveries.

\section{METHODS AND MATERIALS}

\section{A. Phase-space modeling of the linear accelerator}

The BEAMnrc code was used to simulate the "patientindependent" components (target, primary collimator, flattening filter, ion chamber, and mirror) of a Varian 21 EX linear accelerator. The simulation details of our accelerator using BEAMnrc have been described previously ${ }^{15}$ for small field sizes (up to $10 \times 10 \mathrm{~cm}^{2}$ ). The electron-on-target parameters, such as the mean energy of the incident electron and the spatial distribution of the electron beam, were thoroughly analyzed using an iterative procedure and matched with the measured data within 1-2\% accuracy to obtain the optimum values for these variables, especially for larger field sizes. The optimal incident electron beam parameters for this nominal $6 \mathrm{MV}$ photon beam are: energy $=5.9 \mathrm{MeV}$; full width at half maximum (FWHM) of the Gaussian (axis perpendicular to the target) intensity distribution $=0.15 \mathrm{~cm}$. These fixed components are just simulated once and stored in four phase-space (PS) files containing around 200 million particles just above the secondary collimators. The PS files are read into the DPM MC code ${ }^{17}$ and are subsequently used

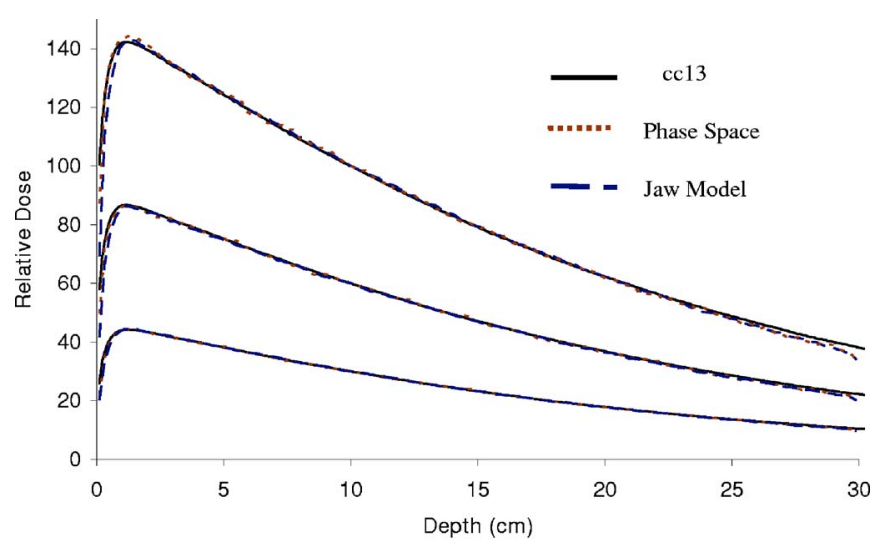

(a)

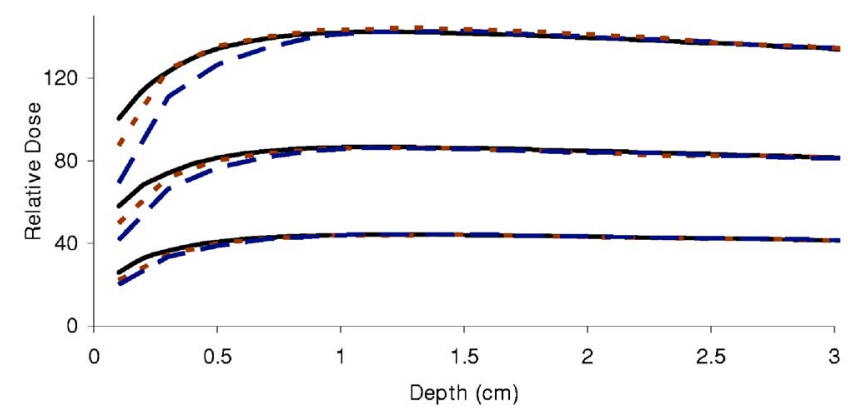

(b)

FIG. 2. Depth dose curves for $6 \mathrm{MV}$ photon beam for field sizes $20 \times 20$, $30 \times 30$, and $40 \times 40 \mathrm{~cm}^{2}$. The graphs compare ion chamber (cc13) measurement with DPM simulations using the PS files and DPM Jaw model, respectively. (b) PDD comparison near buildup region. All the curves are normalized to $10 \mathrm{~cm}$ depth. A multiplication factor of 30, 60, and 100 is applied to $20 \times 20,30 \times 30$, and $40 \times 40 \mathrm{~cm}^{2}$, respectively, to show all the curves on the same graph. The optimum incident electron-on-target parameters for these simulations are: Energy $=5.9 \mathrm{MeV}$; FWHM=0.15 $\mathrm{cm}$.

for the patient-dependent calculations which depend on the MLC, jaws, and patient. Beam modifiers such as jaws and MLC are modeled within the DPM MC code, as shown in Fig. 1. Note that this arrangement of PS files does not account for the backscatter of electrons from the collimators into the monitor chamber in the treatment head. The amount of backscattering into the monitor chamber is higher for smaller field sizes (due to more backscattered particles from the closed collimators) and typically increases by $\sim 2 \%$ as the field size decreases from $40 \times 40$ to $3 \times 3 \mathrm{~cm}^{2}$.

\section{Secondary collimators inside DPM}

Plan-related beam modifiers such as the jaws and MLC have been modeled within DPM using planar geometries and photon-only transport. While multiple photon scattering is accounted for, electrons generated within the collimators deposit their energy locally. The jaw modeling geometry uses planes and is based upon a general strategy for boundarycrossing logic which circumvents ambiguities associated with numerical precision and end-of-step directional uncertainties. ${ }^{19}$ The effect of ignoring electrons in jaws is mainly an issue near the buildup region for larger field sizes. Figure 2 shows the percent depth dose curves (PDDs) for $20 \times 20,30 \times 30$, and $40 \times 40 \mathrm{~cm}^{2}$ field sizes defined by the 


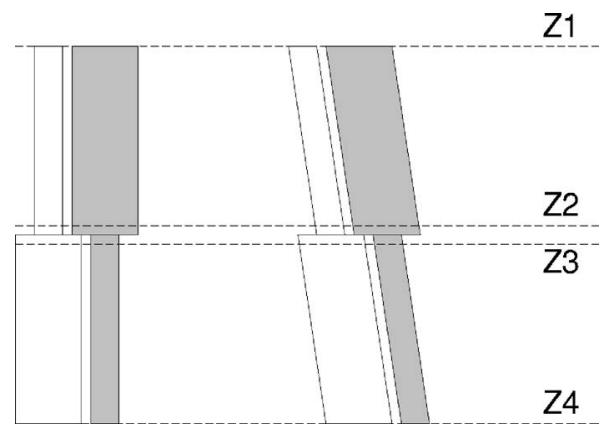

FIG. 3. Leaf geometry details in MLC model.

jaws. All the curves are normalized at $10 \mathrm{~cm}$ depth. The DPM MC dose calculations for these field sizes were performed using (a) full PS simulation: both patient independent components and jaws were simulated using the BEAMnrc code (labeled as Phase Space) for verification purposes and (b) the jaw model in the DPM code (labeled as Jaw Model) using the BEAMnrc-based PS from the patient-independent components as input as shown in Fig. 1. The average $2 \sigma$ uncertainty in the MC calculations was less than $0.5 \%$ at $d_{\max }$ point for all PDDs in this study. The calculations are compared with measurements made with a CC-13 cylindrical ion chamber (Scanditronix, Uppsala, Sweden) with an air cavity volume of $0.13 \mathrm{~cm}^{3}$ and a $3 \mathrm{~mm}$ inner radius. In the buildup dose region $(<0.5 \mathrm{~cm})$, parallel plane chambers were used to verify the doses and adjustments were made where necessary. The agreement between calculations and measurements is within $2 \%$ for these field sizes. A closer look at the buildup region in Fig. 2(b) shows bigger differences versus measurements for calculations with the jaw model as compared to PS calculations at depths below $1 \mathrm{~cm}$. The deviation is greater for the jaw model because the electron transport within the jaw is neglected in the DPM simulations, whereas full electron transport is performed in the BEAMnrc simulations. This shows that ignoring electron transport within the collimators affects the buildup region for larger field sizes for the $6 \mathrm{MV}$ photon beam.

\section{MLC modeling}

A very detailed model of the Varian Millennium MLC within the BEAMnrc MC code has been described by Heath et $a l^{8}$ Since DPM currently does not support a geometry package similar to the BEAMnrc code, we have modeled geometries specific to jaws and the Millennium MLC for IMRT dose calculations. The widths of the leaves were measured at four different planes along the leaf thickness at the MLC plane using a digital micrometer that has an accuracy of $0.0001 \mathrm{in}$. In our source model, we divided the MLC geometry into two regions: top and bottom (see Fig. 3). The variation of the thickness of the leaves between the top and bottom portion of each region has been taken into account, thus differentiating between a target leaf (thicker portion of the leaf towards the target and thinner portion towards the isocenter), an isocenter leaf (thicker portion towards the isocenter and thinner portion towards the target) and a full leaf as shown in Fig. 3.

A plane is formed by joining the points at plane $z 1$ and $z 2$ to form the side edge/plane of each leaf. These leaf sides are thus automatically focused towards the target accounting for divergence along the leaf edges. A similar plane is formed by joining points at plane $z 2$ and $z 3$. The rounded tip for each half is modeled as a part of a cylinder of radius $8.0 \mathrm{~cm}$. The planes forming the sides of each leaf differentiate an individual leaf (with top or bottom region either a target leaf or an isocenter leaf) along with its rounded tip. The rounded tip partially corrects for the divergence and penumbra effect as a function of leaf position.

As each particle is propagated, its position is checked at the top and bottom portion of each half of the leaf to determine which leaf the particle would intersect. This also helps in determining whether the particle is going to intersect the leaf from outside, or is going to emerge from within the leaf or gets attenuated completely inside the leaf or will not intersect at all. Once within the leaf, particles are scattered based on the Compton cross section until they exit from the side of the leaf or from the rounded tip or from the bottom half section of the leaf. For a photon intersecting from the outside, the interaction point is determined by calculating the distance to the rounded tip and side planes for that leaf and, based on the closest distance, it either strikes the side planes or the rounded tip. The particle is moved to the boundary accordingly and the position is then sampled using exponential attenuation

$$
s=-\frac{1}{\mu \rho} \log (\xi),
$$

where $\mu$ is the mass attenuation coefficient in $\mathrm{cm}^{2} / \mathrm{g}$,

$\rho$ is the density of the leaves, and

$\xi$ is a random number in the interval $[0,1)$.

The particle's position is checked at the boundary and updated at each sampled position. The model does not currently account for the $3 \mathrm{~mm}$ driving screw hole; however, we believe this to be a minor issue as the amount of photon attenuation through a $3 \mathrm{~mm}$ air slab is negligible compared to the remaining thickness of the tungsten leaf material. A variable air gap of $0.006-0.008 \mathrm{~cm}$ between the leaves has been used to provide the best agreement between calculations and measured (film-based) leakage profiles. Lower energy photons have a higher probability of undergoing Compton interactions. Multiple Compton scatter is sampled in the MLC until the particle exits the geometry. Electrons generated in the secondary collimators as a result of pair production or photoelectric effects are deposited locally as they constitute a minor fraction of the total fluence. ${ }^{11}$ The electrons that are generated at the bottom of the beam modifiers may contribute to dose in the patient and may be of importance. The effect (on the depth dose curve buildup regions) of neglecting the electrons in the jaws was discussed in the previous section. A similar effect in the buildup region can be expected when electron transport is ignored in the MLC. 


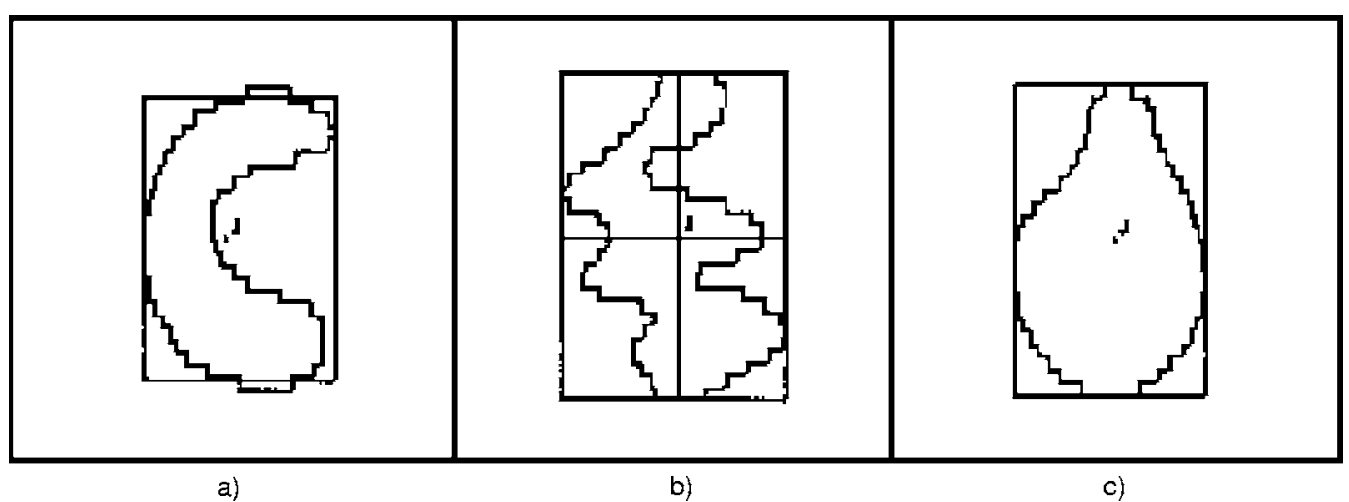

FIG. 4. TG-53 recommended static shapes [reproduced from Fraass et al., Med. Phys. 25, 1773-1829 (1998)].

The cross sections for the tungsten alloy ${ }^{8}(90 \% \mathrm{~W}, 6 \% \mathrm{Ni}$, $2.5 \% \mathrm{Cu}$, and $1.5 \% \mathrm{Fe}$ ) for the MLC are generated within DPM. A density of $16.0 \mathrm{~g} / \mathrm{cm}^{3}$ for the tungsten alloy gives a good estimate of the transmission through the leaves (see below). The cross-section values are obtained using the appropriate function calls for the incident (primary) and the Compton scattered photon. The energy deposition parameter is multiplied with the total monitor units (MUs) for a particular beam and a calibration factor of $0.8 \mathrm{cGy} / \mathrm{MU}$ is applied to calculate the final values in absolute dose (the value of $0.8 \mathrm{cGy} / \mathrm{MU}$ is the calibration factor under the calibration conditions $\left[10 \times 10 \mathrm{~cm}^{2}, 100 \mathrm{~cm}\right.$ source to actual distance, $10 \mathrm{~cm}$ depth in water] used at the University of Michigan for photon beams).

An important consideration regarding the rounded leaf ends is that such a shape causes the projected radiation field, light field, and absolute linear position of the leaves to be different from each other. Hence, a calibration must be applied to match the digital readout of the control system and the radiation field or light field. ${ }^{20}$ Because of this effect, a correction to offset the isocenter leaf locations to their physical positions, based on radiation measurements, is applied to each leaf prior to the simulation.

Our transport model differs from those developed by others ${ }^{10-12}$ in several respects. It transports all photons based on multiple Compton scattering through the MLC geometry. Continuous variation in the thickness of the leaves has been accounted for from the top to the bottom ends of the leaves thereby correctly characterizing leaf divergence in the transverse plane. The model does not transport electrons produced in the jaws or MLC unlike in the component module developed by Heath and Seuntjens. ${ }^{8}$ In addition, we have verified the model using measurements for clinically relevant treatment beams, including beams with high degrees of modulation.

\section{B. SMLC and DMLC implementation within DPM}

DMLC and SMLC delivery methods were simulated by sampling uniformly from a cumulative distribution function (CDF) described by the MU index as a function of leaf segments. The field shapes for each delivery are thus sampled based on the MU index specified in the leaf sequencing file.
For DMLC fields, the leaf positions are uniformly sampled in between the two consecutive leaf positions to simulate the continuous motion profile of the leaves. ${ }^{7}$ The leaf positions can also be sampled based on the actual trajectory of motion (if nonuniform). A random number is generated between 0 and 1 to choose a MU index. The corresponding segment index is selected and the leaves are positioned accordingly. Thus, each MC history for dose calculation first reads a record from the patient independent PS (sequentially), the particles are then transported through the fixed secondary jaws, a monitor unit index is sampled based on the CDF and the particles are further transported through the MLC with leaves positioned based on the corresponding dose segment or the control point (in DMLC). Finally, these particles are incident on the patient geometry and the transport is carried out. (The sequential access of binary files is quicker as a large amount of data are put into the data cache, which is the fastest access. When read randomly, it goes to disk which is much slower.)

The SMLC leaf sequencing algorithm which is studied here is based on Bortfeld's algorithm ${ }^{21}$ implemented with up to 250 dosimetric segments and $1 \%$ dose intervals. ${ }^{22}$ The tongue-and-groove effect is not accounted for in the SMLC leaf sequencing algorithm. The DMLC leaf sequencing algorithm used here, on the other hand, minimizes the tongue and groove by using partial synchronization. There is a forced gap of $1.1 \mathrm{~mm}$ between the opposed leaves to account for delivery constraints. ${ }^{22}$

\section{Measurement and phantom setup}

Film measurements were performed with Kodak EDR (extended dose range) film in a homogeneous solid water (Gammex RMI, Middleton WI) phantom of dimensions 30 $\times 30 \times 30 \mathrm{~cm}^{3}$ at $5 \mathrm{~cm}$ depth, $95 \mathrm{~cm} \mathrm{SSD}$ for a $6 \mathrm{MV}$ photon beam. The films were exposed at a dose rate of $400 \mathrm{MU} / \mathrm{min}$. A dose calibration curve was measured at the same time and used to convert the film response from optical density to dose. All the processed films were digitized using a laser film digitizer (Lumisys Lumiscan LS75, Kodak, Rochester, NY). The images were analyzed using IGOR pro software (Interactive Graphics Oriented Research) programmed to recognize and read the Lumisys file formats. 
The software has tools to plot one-dimensional line profiles, two-dimensional contour plots, dose difference displays, and histogram distributions. In addition, a dose-gradient analysis tool (called the gradient compensation method described in detail by Moran et al. $)^{23}$ has been implemented within IGOR to analyze the discrepancy in dose difference between calculation and measurement. This method evaluates local dosimetric differences as a function of the dose gradient at each point in the dose distribution and thus highlights effects that are significant in the dose difference map not due to any geometric misalignments. A distance parameter, which is typically the size of geometric uncertainty $(1 \mathrm{~mm}$ in all our examples), is chosen and the dose difference, which could be caused by a geometric shift of this distance, is removed from the dose-difference distribution by the gradient compensation in order to highlight differences which could not be explained by the specified geometric shift. An IC-10 (Scanditronix, Uppsala, Sweden) cylindrical ion chamber with an air cavity volume of $0.13 \mathrm{~cm}^{3}$ and a $3 \mathrm{~mm}$ inner radius was also used to measure dose in a Scanditronix/Wellhöfer water scanning system for MLC static shapes at various depths. The verification measurements are described below.

\section{MLC leakage measurement}

Inter-leaf and intra-leaf leakage was measured using a $10 \times 30 \mathrm{~cm}^{2}$ field defined by jaws, with the MLC leaves blocking the entire open field. The tips of these leaves were $2 \mathrm{~cm}$ beneath/behind the jaws. A fluence of $5000 \mathrm{MU}$ was delivered for this field. Because of the smaller film size used for the measurement, the beam was delivered on two films by dividing the field size into: $y 1=0 \mathrm{~cm}, y 2=15 \mathrm{~cm}$ and $y 1=15 \mathrm{~cm}$, and $y 2=0 \mathrm{~cm}$ with the $\mathrm{x}$ jaws defining a $10 \mathrm{~cm}$ field. A $10 \times 10 \mathrm{~cm}^{2}$ open field was also exposed with $100 \mathrm{MU}$ to determine the open field dose in order to estimate the percent transmission. The same $10 \times 10 \mathrm{~cm}^{2}$ jaw positions were used to measure leakage from the closed-leaf tip (with the leaf tip located on the central axis) by delivering $400 \mathrm{MU}$.

\section{MLC leaf positioning-alternate even and odd leaf pattern}

MLC leaf widths/positions were checked by exposing films to $100 \mathrm{MU}$ with the jaws defining a field size of 10 $\times 30 \mathrm{~cm}^{2}$ at $5 \mathrm{~cm}$ depth, $95 \mathrm{~cm} \mathrm{SSD}$. MLC leaves were placed in two different alternate configurations-(a) even leaves closed and beneath the jaws and odd leaves open making a width of $10 \mathrm{~cm}$ along the leaf direction, and (b) odd leaves closed and beneath the jaws and even leaves open. Again, because of the smaller film size used for the measurement, the field size was divided into two: $y 1=0 \mathrm{~cm}, y 2$ $=15 \mathrm{~cm}$ and $y 1=15 \mathrm{~cm}$, and $y 2=0 \mathrm{~cm}$ with $x$ jaws defining a $10 \mathrm{~cm}$ field.

\section{MLC arbitrary shaped static shapes}

Dose profiles were measured with an ion chamber (IC-10) for arbitrary static field examples, labeled "M5," "M6," and "M7" (Fig. 4). These example field shapes have been sug-
TABLE I. Monitor units and number of segments for SMLC and DMLC beams.

\begin{tabular}{ccccc}
\hline \hline Plan & $\begin{array}{c}\text { MLC } \\
\text { delivery }\end{array}$ & $\begin{array}{c}\text { Monitor } \\
\text { units }\end{array}$ & Segments & $\begin{array}{c}\text { Field size } \\
\left(\mathrm{cm}^{2}\right)\end{array}$ \\
\hline Partial breast & SMLC & 345 & 222 & $5 \times 8$ \\
Partial breast & DMLC & 375 & 150 & $5 \times 8$ \\
Prostate & SMLC & 120.4 & 180 & $8.5 \times 7.5$ \\
Head and neck & SMLC & $119+73$ & $290+190$ & $8.7 \times 14.9$ \\
\hline \hline
\end{tabular}

gested for testing the MLC beam model in the American association of physicists in medicine (AAPM) Task Group Report No. $53 .{ }^{24}$ The jaw positions for these fields were 22 $\times 29,22 \times 29$, and $23 \times 35 \mathrm{~cm}^{2}$, respectively, at $90 \mathrm{~cm} \mathrm{SSD,}$ $10 \mathrm{~cm}$ depth. The profiles were measured at depths of 3, 5, 10 , and $20 \mathrm{~cm}$ for all the shapes along the transverse and radial planes. The PDD for the "M6" shape is measured at an off-axis point $(-4,0)$ which is also the center of the $\mathrm{C}$ shape.

\section{SMLC and DMLC validation}

Measurements were performed for SMLC and DMLCsequenced plans for anatomical sites including the breast, prostate, and head and neck. All verification measurements in this study were performed with the gantry perpendicular to the phantom and film (i.e., AP orientation only). EDR films for the measurements were embedded in a homogeneous solid water phantom of size $30 \times 30 \times 30 \mathrm{~cm}^{3}$ at $5 \mathrm{~cm}$ depth, $95 \mathrm{~cm}$ SSD. Table I shows the monitor units, jaw positions, and number of segments delivered for these beams. The head and neck beam chosen for this study is split into two fields with very high modulation since the maximum field width obtainable using a Varian 120 MLC leaf collimator is $14.5 \mathrm{~cm}$. For field sizes larger than this, the sequencer splits the fields into two individual fields. All the beams are optimized and sequenced with $1 \times 1 \mathrm{~cm}^{2}$ beamlets except the prostate beam that uses $0.5 \times 0.5 \mathrm{~cm}^{2} \mathrm{~cm}$ beamlets. The prostate beam was sequenced for a protocol designed to spare the urethra and hence demanded a very high degree of modulation.

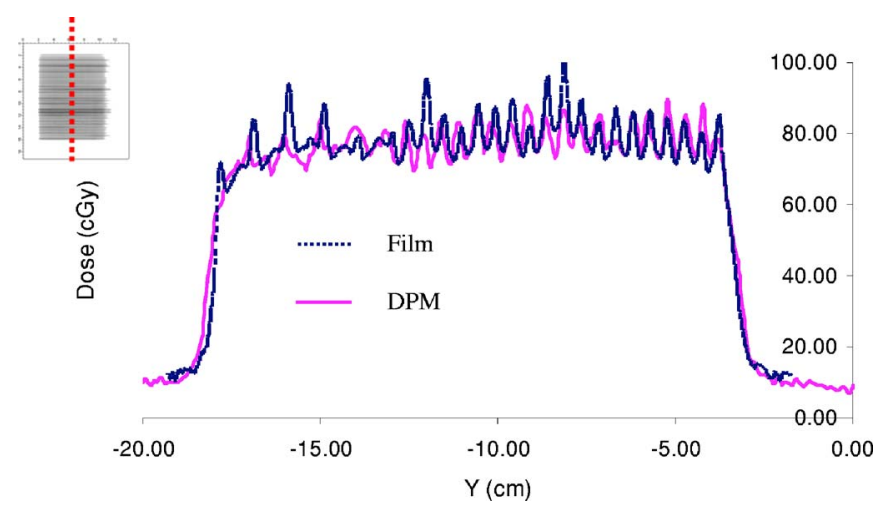

FIG. 5. Leakage pattern through the top $25(y 1=0 ; y 2=15 \mathrm{~cm})$ closed leaves forming a $10 \times 30 \mathrm{~cm}^{2}$ field size; $5000 \mathrm{MU}$ were delivered for this pattern. 


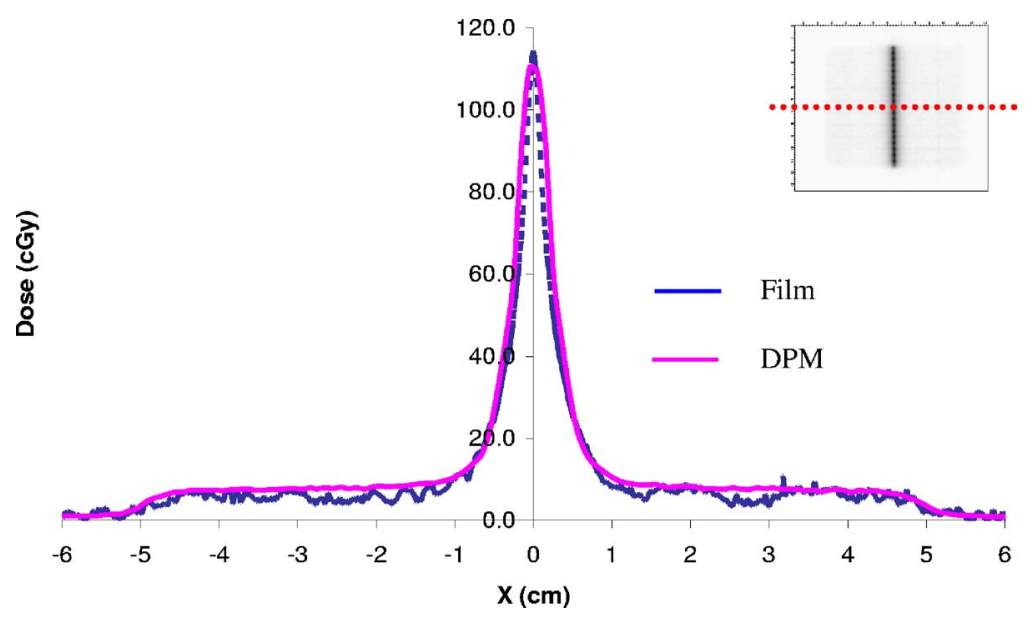

FIG. 6. Leakage pattern through the closed leaf tips forming a $10 \times 10 \mathrm{~cm}^{2}$ field size; $400 \mathrm{MU}$ were delivered for this pattern.

\section{Monte Carlo calculations}

DPM calculations were performed with a $1 \mathrm{~mm}$ step size, and low energy electron and photon cutoffs of 200 and $50 \mathrm{keV}$, respectively. All calculations are reported in absolute dose (cGy) using the following equation:

$$
\operatorname{Dose}_{\mathrm{FS}}(\mathrm{cGy})=\frac{\operatorname{Dose}_{\mathrm{FS}}(\mathrm{MeV} \cdot \mathrm{MU} / \mathrm{g})}{\operatorname{Dose}_{10 \times 10}(\mathrm{MeV} / \mathrm{g})} \times 0.8(\mathrm{cGy} / \mathrm{MU})
$$

where, $\operatorname{Dose}_{\mathrm{FS}}(\mathrm{cGy})=$ Dose in cGy for any field size

$$
\begin{aligned}
\text { Dose }_{10 \times 10}(\mathrm{MeV} / \mathrm{g})= & \text { Dose in } \mathrm{MeV} / \mathrm{g} \\
& \text { for a } 10 \times 10 \mathrm{~cm}^{2} \text { field size }
\end{aligned}
$$

$\operatorname{Dose}_{\mathrm{FS}}(\mathrm{MeV} . \mathrm{MU} / \mathrm{g})=$ Dose in $(\mathrm{MeV} \cdot \mathrm{MU}) / \mathrm{g}$

$$
\text { for any field size }
$$

and $0.8 \mathrm{cGy} / \mathrm{MU}$ is the calibration factor applied at the University of Michigan.

Plans were generated using DPM calculations in a homogeneous water phantom of $25.6 \times 25.6 \times 30 \mathrm{~cm}^{3}$ dimensions and voxel sizes $1-2 \mathrm{~mm}$ for the leakage, leaf position analysis, and IMRT calculations. A larger phantom $(40 \times 40$ $\left.\times 40 \mathrm{~cm}^{3}\right)$ with slightly larger voxel sizes $(5 \mathrm{~mm})$ was used for the arbitrary shaped fields. Sufficient histories $(\sim 10-20$ billion) were simulated for each run such that the uncertainty $(1 \sigma)$ of the average dose over all voxels greater than Dose $_{\max } / 2$ was less than $1 \%$, unless otherwise stated. The calculations were run in parallel on cluster of AMD Athlon processors $(2.4 \mathrm{GHz})$ using a 64 bit Linux architecture. Each IMRT beam required $\sim 8 \mathrm{~h}$ of simulation time (transport through the jaws, MLC and phantom) running on eight processors.

\section{RESULTS AND DISCUSSION}

\section{A. MLC leakage measurement}

Figure 5 shows the comparison between DPM calculations and EDR film for the top 25 leaves (for a field size of $10 \times 30 \mathrm{~cm}^{2}$ defined by the jaws). The voxel size used for this calculation was $0.5 \times 0.1 \times 1 \mathrm{~cm}^{3}(0.1 \mathrm{~cm}$ in the direc- tion across the leaf gaps). The film data were measured twice on different days (with a gap of two weeks) and the reproducibility for the leakage test was within $2 \%$. Similar observations were found for the bottom 25 leaves. The MLC model is able to reproduce the peaks and valleys of the film data. There are slight differences due to machine tolerance and reproducibility of the leaf positions as well as uncertainty in DPM calculation (which is approximately 3\% in the peaks). The dose ( $\sim 90-100 \mathrm{cGy})$ shown in Fig. 5 actually represents only $1.5-2 \%$ of the total open dose (compared to an open $10 \times 10 \mathrm{~cm}^{2}$ field size) and is referred to as the percent transmission or the leakage through the closed leaves.

The leakage through the rounded leaf tip was also calculated using $400 \mathrm{MU}$ for a voxel size of $1 \mathrm{~mm}$ along the tip for the DPM calculation. Figure 6 shows the comparison between the film data and DPM calculation and shows that the model reproduces the leaf-tip curvature accurately. The dose represented here $(\sim 110 \mathrm{cGy})$ amounts to roughly $25 \%$ of the open field $\left(10 \times 10 \mathrm{~cm}^{2}\right)$ dose. In the tail region, the calculation appears to overestimate the film data. However, the accuracy of the film and calculations are difficult to interpret in this region. The film dose is limited by noise and its energy dependence. ${ }^{25}$ The DPM calculations are limited by a larger statistical uncertainty.

\section{B. MLC alternate even and odd leaf pattern}

The MLC alternate even and odd leaf pattern test was used to verify the accuracy of the modeled leaf widths. The MLC leaves were placed in two different configurations. Figure 7 shows the case where the top 25 even leaves are closed and beneath the jaws and the odd leaves are open. The agreement between film and DPM calculation is within $1 \%$ in the high dose regions and within $1 \mathrm{~mm}$ in the high gradient regions. The percent error in DPM calculation in all the peaks is approximately $1.5 \%$ and the error associated with film measurement is approximately $2 \%$. Considering these two sources of uncertainty, DPM agrees very well with the measurements. Similar results were found for the bottom 25 alternate even and odd leaf patterns. 


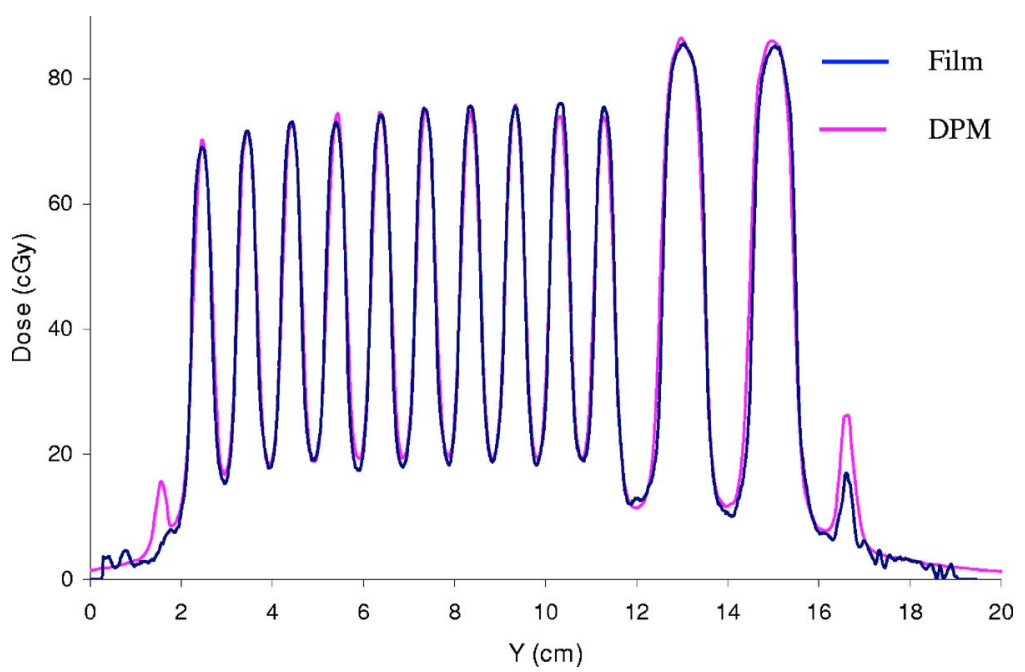

FIG. 7. Leaf positioning test for alternate leaf pattern with even leaves closed and odd leaves open for the top 25 leaves $(y 1=0 ; y 2=15 \mathrm{~cm})$.

\section{MLC arbitrary shaped static shapes}

Static shapes that are part of our regular commissioning dataset and suggested in AAPM Task Group Report No. 53-M5 $\left(22 \times 29 \mathrm{~cm}^{2}\right), \quad$ M6 $\left(22 \times 29 \mathrm{~cm}^{2}\right)$ and M7 $(23$ $\times 35 \mathrm{~cm}^{2}$ ) were simulated and compared with the measured ion chamber (IC-10) PDDs and profiles. The voxel size used for these sets of calculation is $0.5 \times 0.5 \times 0.5 \mathrm{~cm}^{3}$. Figure 8 shows excellent agreement (less than $2 \%$ ) at regions beyond the dose buildup between the calculated and measured PDDs for these arbitrary static shapes. In addition, output factors (OFs) were calculated and compared with the measured OFs. The PDD and OF for the "M6" shape were evaluated at the center of the C shape $(-4,0)$. Table II shows that OFs calculated using the MLC model are within $2 \%$ of the measured OFs. These slight discrepancies are caused by three main factors: the effect of backscattered particles in the monitor chamber that is not accounted for in the simulations, uncertainty in DPM simulation (less than 0.5\%) and measurement reproducibility $(\sim 1 \%)$. The OFs shown in Table II are corrected using the measured backscatter fraction for an equivalent square field. ${ }^{18}$

Transverse profiles and radial profiles were also extracted along the central axis at various depths: $3,5,10$, and $20 \mathrm{~cm}$.

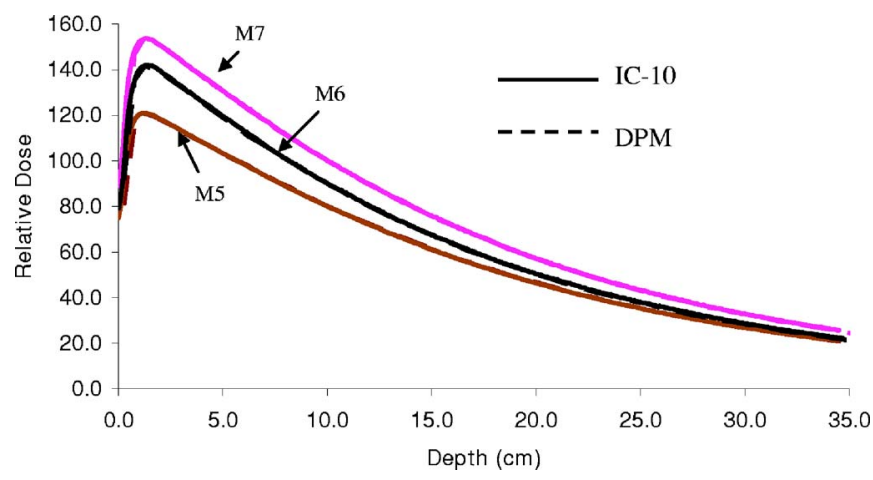

FIG. 8. Depth dose curve comparison between ion chamber measurement and DPM calculation for the AAPM TG-53 recommended static shapes: M5, M6, and M7. The curves are normalized at $10 \mathrm{~cm}$ depth.
Figures 9(a) and 9(b) show the transverse profiles and radial profiles, respectively, for the "M7" shape along the lines shown in the inset. The transverse profiles for the M7 shape agree very well with the ion chamber data. The radial profiles, however, show extra leakage ( $\sim 25 \%$ at $10 \mathrm{~cm}$ depth) outside the open field. These profiles are extracted along the central axis. The jaws for these fields are wider than the MLC field and the central axis passes through the leaf tips of the opposed leaves. The leakage from the rounded tip of the MLC leaves accounts for the extra leakage of approximately $25 \%$.

The good agreement between calculations and ion chamber measurements in the penumbral regions may be due to the fact that the large voxel sizes used in these calculations $\left(0.5 \times 0.5 \times 0.5 \mathrm{~cm}^{3}\right)$ and hence larger volume averaging effects are of equal magnitude to the volume averaging of dose within the ion chamber. The profiles are also within error bars in the valley for the M7 shape where there is more pronounced volume averaging of dose within the ion chamber.

\section{SMLC and DMLC Comparisons}

\section{Partial breast IMRT beam: SMLC and DMLC delivery}

A SMLC IMRT beam for a partial breast treatment was delivered with 345 monitor units and 222 segments. Figure 10 shows comparisons between DPM simulations and film measurements in a homogeneous phantom at $5 \mathrm{~cm}$ depth,

TABLE II. Output factors for the TG-53 recommended MLC static shapes.

\begin{tabular}{|c|c|c|c|c|}
\hline & $\begin{array}{c}\text { Measured } \\
\text { OF }\end{array}$ & $\begin{array}{l}\text { Measured } \\
\text { BSF (for } \\
\text { equivalent } \\
\text { square field) }\end{array}$ & $\begin{array}{c}\text { Calculated OF } \\
\text { corrected for } \\
\text { BSF }\end{array}$ & $\%$ difference \\
\hline M5 & 1.084 & 1.008 & 1.075 & 0.87 \\
\hline M6 (-4.0) & 1.036 & 1.008 & 1.051 & -1.48 \\
\hline M7 & 1.058 & 1.0095 & 1.047 & 1.054 \\
\hline
\end{tabular}




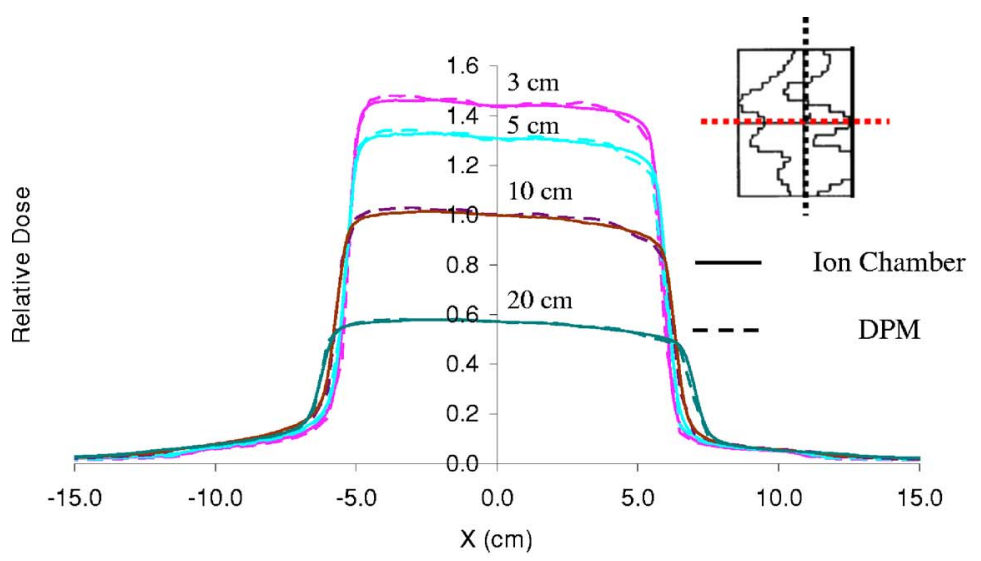

(a)

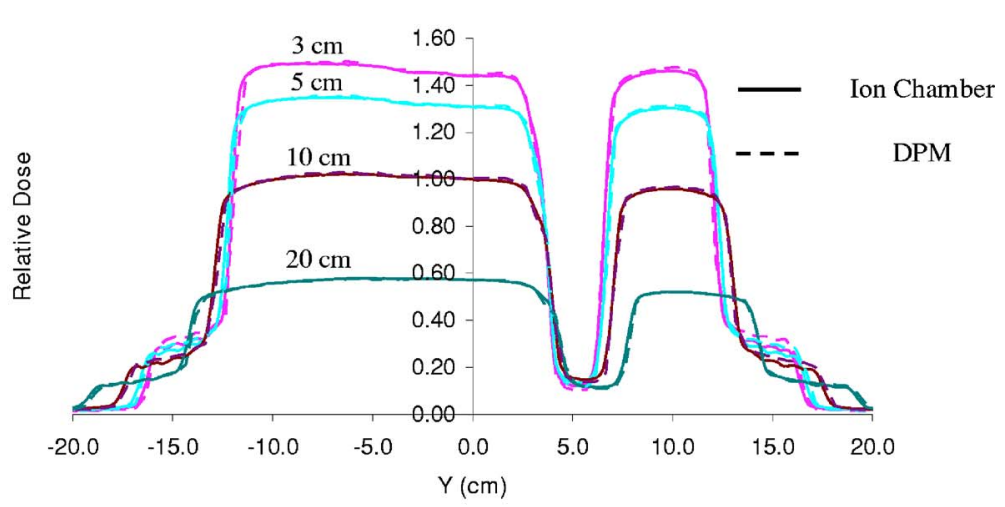

(b)
FIG. 9. Comparison between ion chamber measurement and DPM calculation for a Task Group Report No. 53 recommended static shape (M7). The profiles are extracted at the depths of $3,5,10$, and $20 \mathrm{~cm}$ along the lines shown in the inset. (a) Transverse profiles, (b) radial profiles.
$95 \mathrm{~cm}$ SSD. Figure 10(a) is the intensity map delivered for this beam. Isodose line comparison from 20 to $220 \mathrm{cGy}$ of the measured (film) and calculated (DPM) dose is shown in Fig. 10(b). DPM calculated isodose lines (shown using dotted lines) are in excellent agreement with the film (shown using solid lines) for all curves. The agreement with the film data is within 2-3 mm; a few larger differences were noted at locations where both the film and the DPM calculated doses are limited by noise (low dose regions). Profiles extracted along the radial and transverse direction (on the intensity map) are shown in Fig. 10(c). The agreement between film and DPM calculation in these profiles is in good agreement (less than 2\%) especially in the tongue and groove region (shown in the blue line profile). The $200 \mathrm{cGy}$ isodose line also exhibits this tongue and groove under dosage and shows how accurately DPM is able to reproduce those regions that depend on the details of the MLC model. Figure 10(d) shows the dose difference map between film and DPM simulation (DPM-film). The dose difference map shows differences of up to $30 \mathrm{cGy}$ with the average difference around -1.8 cGy. Most of these differences are in the outer penumbra region. One possible reason for these differences is the much larger voxel size resolution of the DPM calculation $(2 \mathrm{~mm})$ as compared with film $(0.1 \mathrm{~mm})$. This could result from a misalignment in the high dose gradient regions. In the penumbra region, a shift of $1 \mathrm{~mm}$ can result in a dose difference of more than $10 \%$. In the out-of-field regions for the IMRT beams, the film over responds due to the low-energy scattered photons. ${ }^{26}$ When a $1 \mathrm{~mm}$ of gradient compensation is applied [Fig. 10(e)], many of the regions of large dose difference disappear. This shows that most of these differences are due to geometrical errors of less than $1 \mathrm{~mm}$. One beamlet shows a difference of $6 \mathrm{cGy}$ (or 2.4\%) where the laser film digitizer (Lumysis) saturates the film at $3 \mathrm{OD}$. The film doses are therefore flat in this region and the MC doses are higher. The dose difference histograms of the dose difference map and the gradient compensated dose difference map are shown in Fig. 10(f). The relatively large difference of up to 30 cGy shown by the long tails (dotted curve) in Fig. 10(f) is associated with pixels in the high dose gradient and the out-of-field regions of the dose difference map [Fig. 10(d)]. The dose difference histogram of the gradient compensated map (solid curve), however, shows a very narrow peak.

The same partial breast IMRT field was sequenced and delivered using partial leaf synchronization for DMLC delivery. This sequence required 375 monitor units and consisted of 150 DMLC segments. Coronal isodose lines and profiles are shown in Figs. 11(b) and 11(c). Isodose lines from 20 to $240 \mathrm{cGy}$ are in excellent agreement except for the 200 cGy isodose line where the film data are limited by noise. Profiles extracted along the radial and transverse direction are shown in Fig. 11(c). The agreement between film and calculation in these profiles is excellent (less than $2 \%$ ). Note that there is no tongue and groove region in the DMLC IMRT beam due to partial synchronization of the leaves 


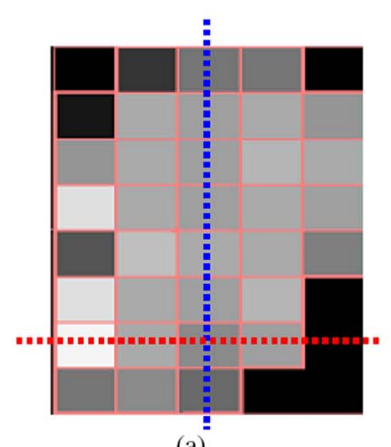

(a)
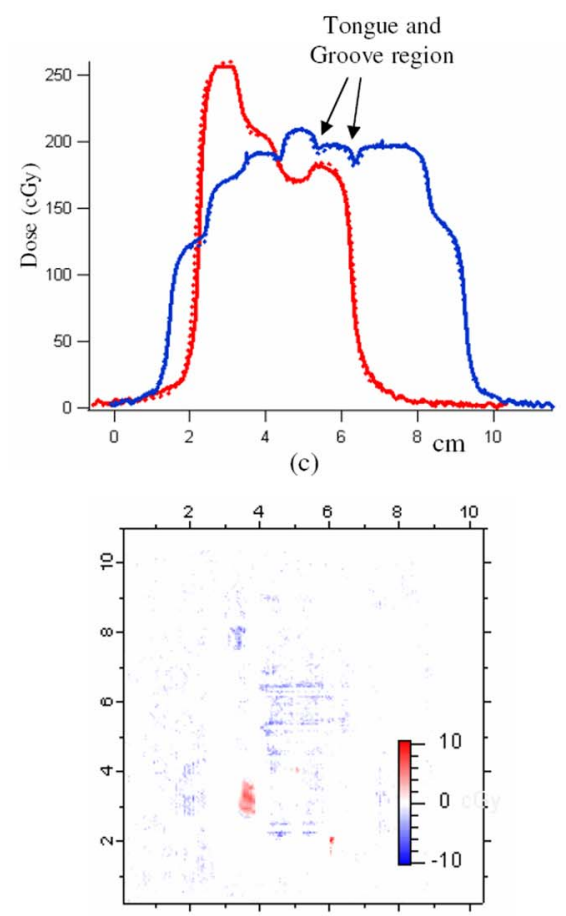

(e)

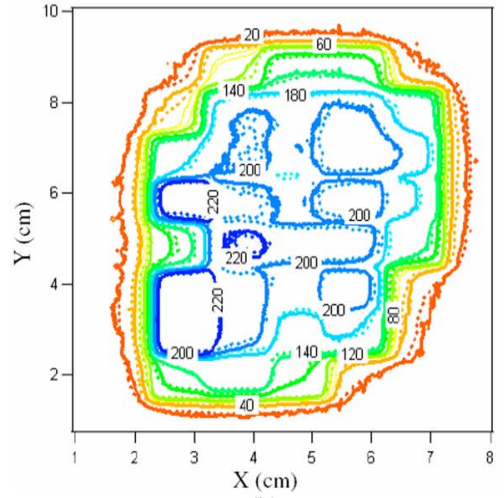

(b)

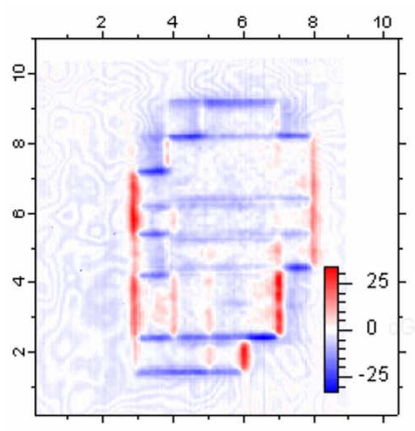

(d)

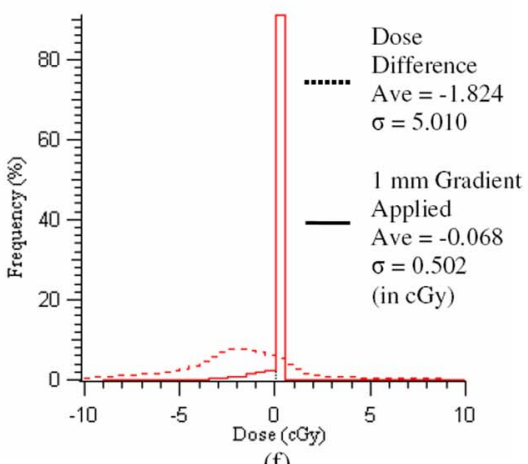

(f)
FIG. 10. Breast IMRT beam simulated using SMLC with 345 MUs and 222 segments. (a) Beam intensity map, (b) isodose display for film measurement and DPM calculation; film is shown in solid and DPM in dashed lines. (c) One-dimensional profile comparisons between film measurement and DPM calculation; film is shown in solid and DPM in dashed lines. (d) Dose difference map in cGy: (DPM-film). (e) Dose difference map (in cGy) generated by applying a $1 \mathrm{~mm}$ gradient compensation. (f) Dose difference histogram of the dose difference map (dotted line) and the gradient compensated dose difference map (solid line). which minimizes tongue and groove effects. Figure 11(d) shows the dose difference map between film and DPM calculation. Within the field, the agreement versus measurement is within $2 \%$. The largest differences of up to $30 \mathrm{cGy}(\sim 12 \%$ of the maximum dose) are again seen in the outer penumbral regions where a difference in dose-grid resolution between film and calculation could result in poor alignment causing a shift of $1 \mathrm{~mm}$ or more in distance to agreements. Dose differences analyzed using $1 \mathrm{~mm}$ of gradient compensation [Fig. 11(e)] shows differences that are not due to geometrical misalignment between film and calculation grid. Similar differences (as in SMLC) are seen in the beamlet saturated by the laser film digitizer.

\section{Prostate}

A prostate IMRT beam was sequenced with 0.5 $\times 0.5 \mathrm{~cm}^{2}$ beamlets using $120 \mathrm{MU}$ and 184 SMLC segments. Figure 12(a) shows the intensities and Fig. 12(b) shows the isodose lines from 5 to $45 \mathrm{cGy}$. The agreement between film and calculation is within $2 \mathrm{~mm}$ for all the iso- dose lines except for the $45 \mathrm{cGy}$ line which lies within the tongue-and-groove regions. Figure 12(c) shows the profile along the directions shown on the intensity map. The horizontal profile shows a larger difference between measurement and calculations which may be due to the rounded leaftip model which uses an untapered cylindrical geometry along the radial plane for the leaf-tip design. This would cause additional attenuation through the leaf tip along the radial plane. Figure 12(d) shows the dose difference map between film and DPM calculation. The differences lie within \pm 6 cGy $(\sim 12 \%$ of the maximum dose). When $1 \mathrm{~mm}$ of gradient compensation is applied, most of the large differences disappear except for a few regions of tongue and groove and penumbral misalignment [Fig. 12(e)].

\section{Head and neck}

A split-field AP beam from an example head and neck treatment plan was sequenced for SMLC. The sequence consisted of 290 segments and required 119 MUs for the first field and 190 segments and 73 MUs for the second field. 


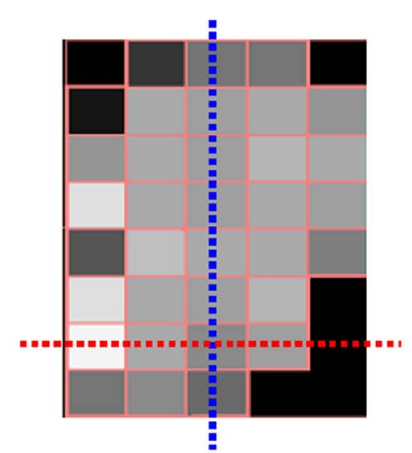

(a)

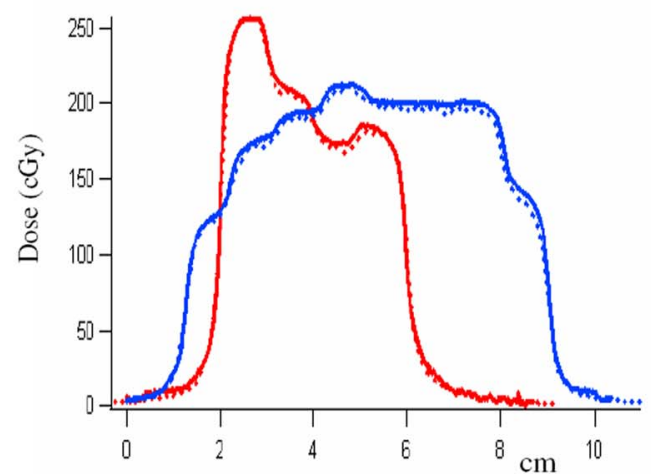

(c)

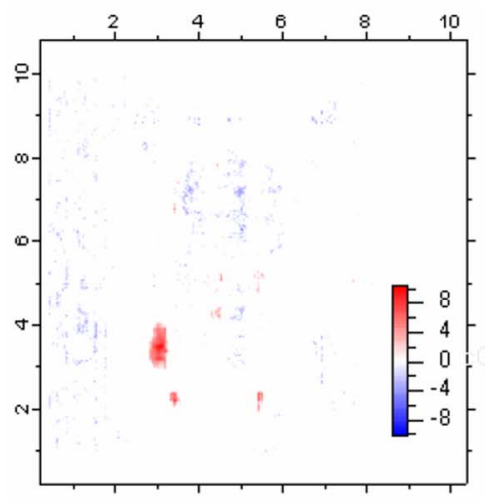

(e)

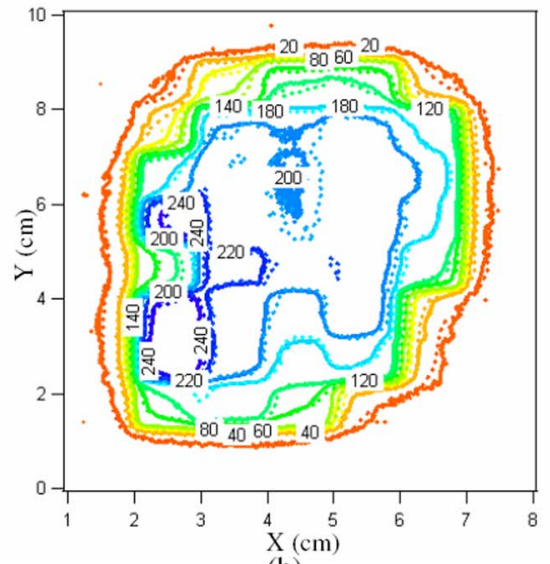

(b)

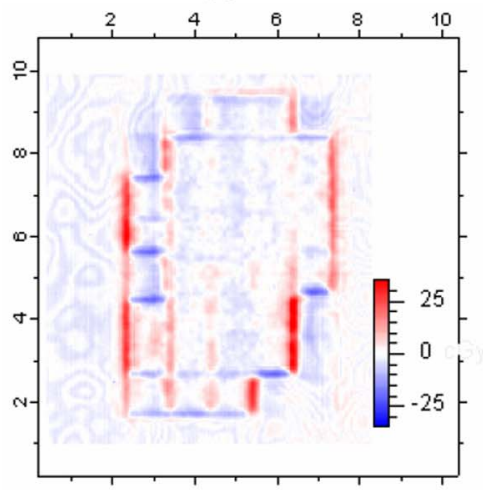

(d)

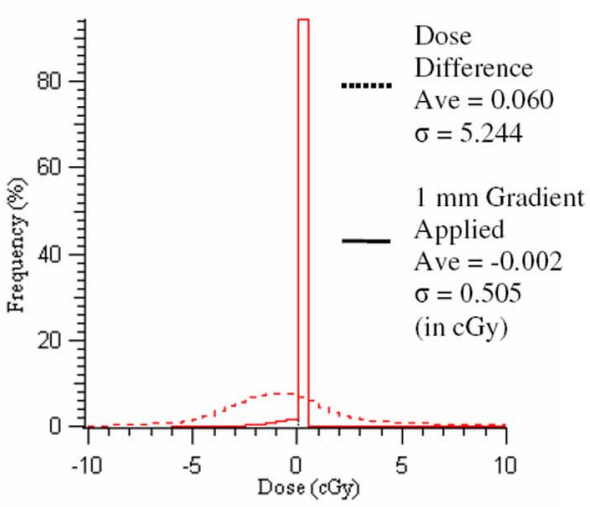

(f)
FIG. 11. Breast IMRT beam simulated using DMLC with 375 MUs and 150 segments. (a) Beam intensity map. (b) Isodose display for film measurement and DPM calculation; film is shown in solid and DPM in dashed lines. (c) One-dimensional Profile comparisons between film measurement and DPM calculation; film is shown in solid and DPM in dashed lines. (d) Dose difference map in cGy: (DPM-film) (e) Dose difference map (in cGy) generated by applying a $1 \mathrm{~mm}$ gradient compensation. (f) Dose difference histogram of the dose difference map (dotted line) and the gradient compensated dose difference map (solid line).
Figure 13(a) shows the intensity map and Fig. 12(b) shows the isodose lines from 10 to $55 \mathrm{cGy}$. Overall, the agreement between these isodose lines is excellent. Some differences are seen in the $20 \mathrm{cGy}$ isodose lines which are likely due to the energy response of the film. Profiles were extracted along the transverse and radial direction [Fig. 13(c)] for both film and DPM simulation. Figure 13(d) shows the dose difference map between film and DPM calculation. The largest differences are within $\pm 8 \mathrm{cGy}(\sim 13 \%$ of the maximum dose $)$ and occur in the high gradient regions. The combined field is very highly modulated and is a stringent test of the MLC model's ability to accurately simulate IMRT beams. The largest differences appear in the field overlap regions. With a $1 \mathrm{~mm}$ gradient compensation [Fig. 13(e)] the regions of geometric misalignment disappear. Differences of the order of
2-3 cGy (up to $5 \%$ of the maximum dose) can still be seen in some high dose regions. Some of these differences may be attributed to the backscattered electrons into the monitor chamber that are not accounted for in the DPM simulation, or may be due to uncertainties in the measurements and/or statistical fluctuations in the MC calculation. Again, the dose difference histograms of the dose difference map and gradient compensated dose difference map are evaluated in Fig. 13(f).

\section{CONCLUSIONS}

In this study we have, through extensive verification, shown that our DPM-based MLC geometry model is capable of accurately modeling the Varian Millennium (120-leaf) 


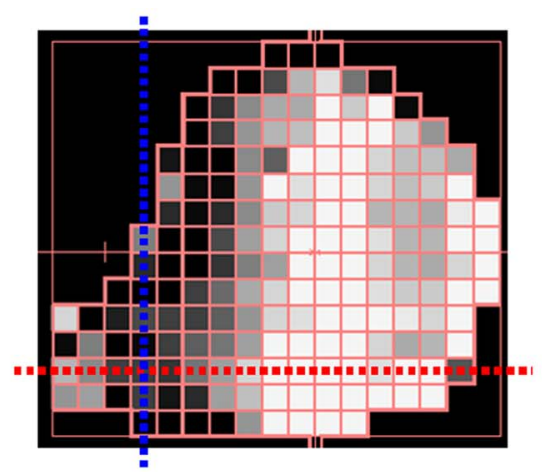

(a)

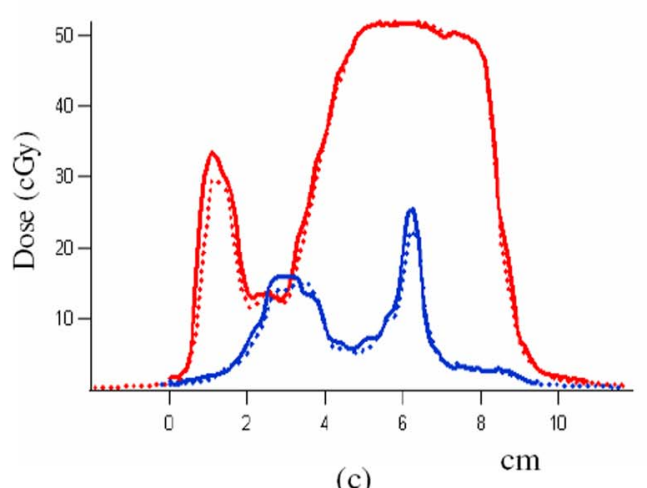

(c)

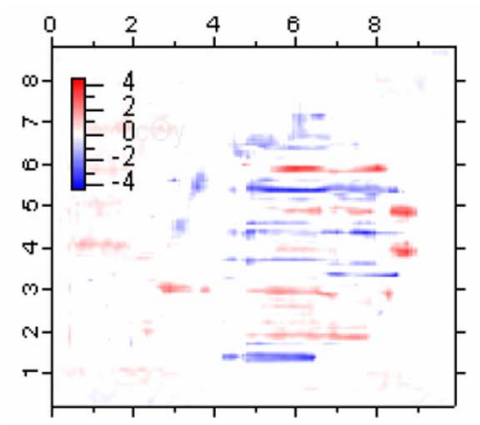

(e)

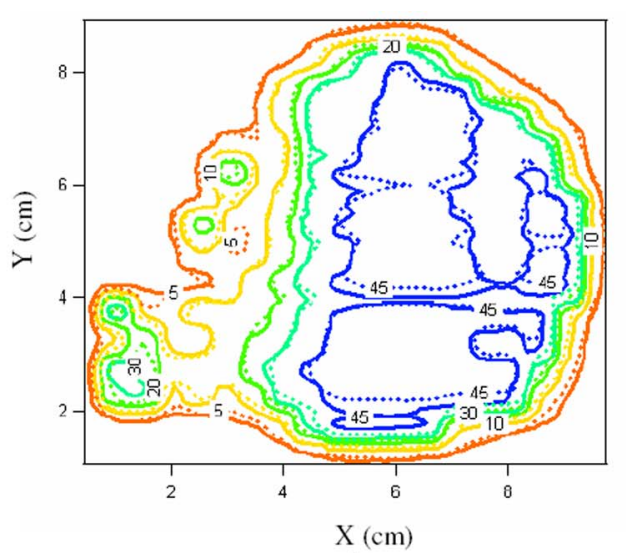

(b)

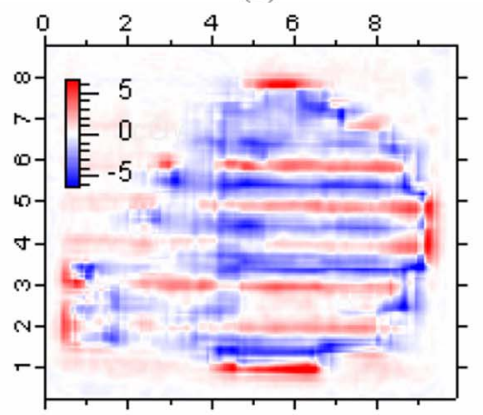

(d)

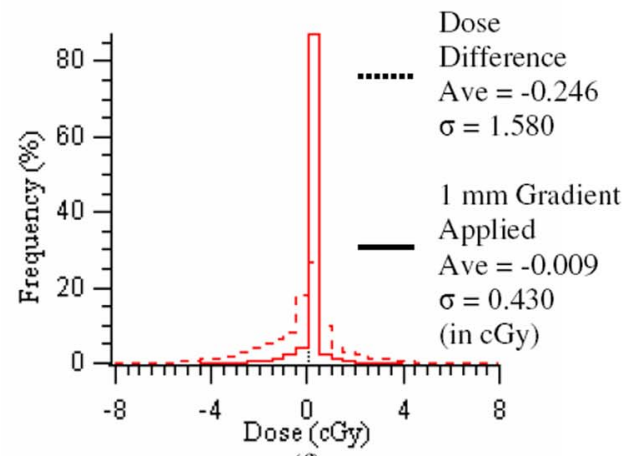

(f)

FIG. 12. Prostate IMRT beam simulated using SMLC with 120.4 MUs and 180 segments. (a) Beam intensity map. (b) Isodose display for film measurement and DPM calculation; film is shown in solid and DPM in dashed lines. (c) One-dimensional profile comparisons between film measurement and DPM calculation; film is shown in solid and DPM in dashed lines. (d) Dose difference map in cGy: (DPM-film). (e) Dose difference map (in cGy) generated by applying a $1 \mathrm{~mm}$ gradient compensation. (f) Dose difference histogram of the dose difference map (dotted line) and the gradient compensated dose difference map (solid line).

MLC and collimating jaws for IMRT dose calculations. Leakage, leaf positioning and static MLC shape tests were performed to verify the details of the MLC model. IMRT beams from three clinical treatment plans for partial breast (both SMLC and DMLC), prostate (SMLC) and head and neck split field (SMLC) were simulated and compared with the film measurements.

For plans that require the MLC leaves to be blocked for a significant fraction of monitor units, total transmission dose can form a significant fraction of the total dose. Leakage through the MLC for the Varian machine accounts for $\sim(1.5-2) \%$ of the total open field dose, so accurate modeling of penumbra and transmission through these leaves can be quite significant since IMRT beams are delivered as a superposition of small fields by moving the MLC leaves through the irradiated area. The MLC model described here is able to reproduce effects due to leaf geometry such as leakage, tongue and groove and rounded tip quite accurately. The good agreement between calculations and measurements for static arbitrary shapes and IMRT beams from several different anatomical sites provides good initial support for the use of this model as a tool for IMRT verification.

Future work will focus on incorporating an electron source to better model the buildup region, and a parameterization to account for the backscattered electrons from the jaws to the monitor chamber. In addition, we will further investigate the accuracy of the IMRT model in heterogeneous media. The eventual goal of this work is to complete 


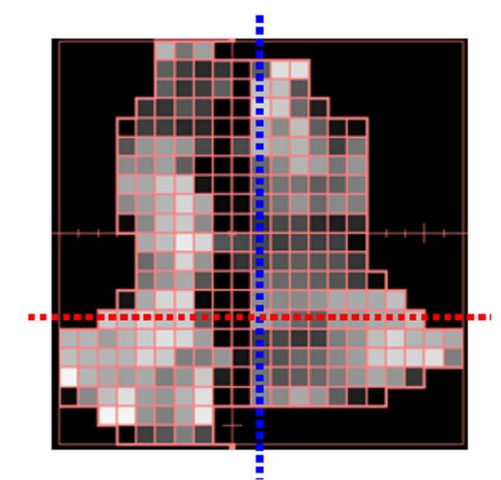

(a)

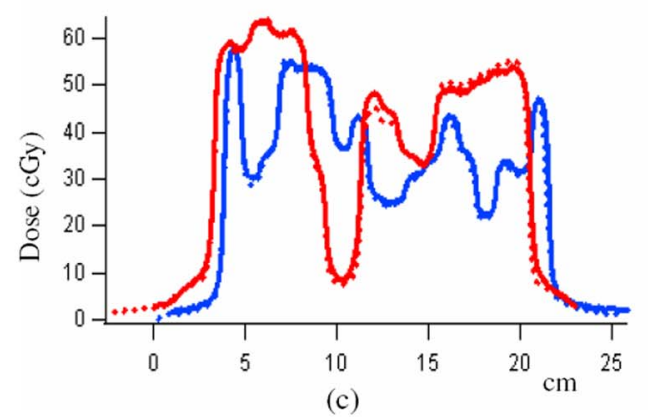

(c)

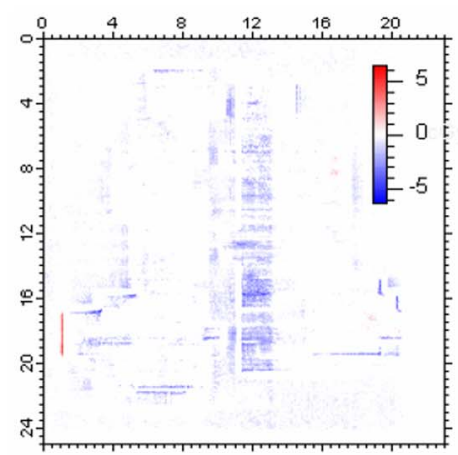

(e)

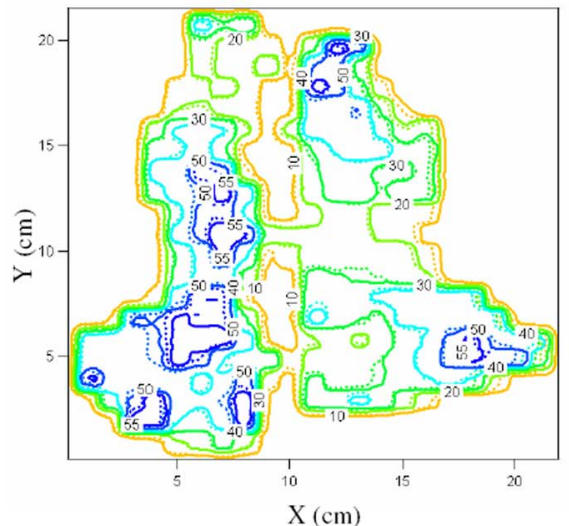

(b)

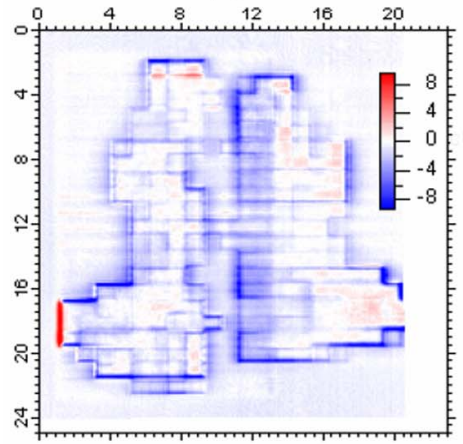

(d)

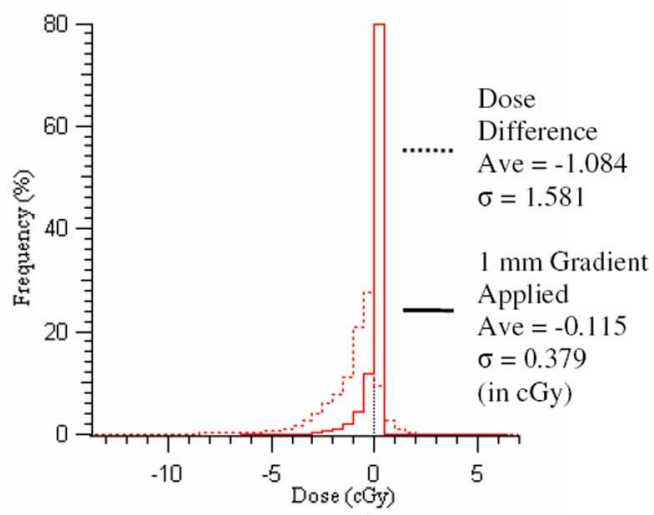

(f)

FIG. 13. Head and neck IMRT beam (split field) simulated using SMLC with $(119+73)$ MUs and $(290+190)$ segments. (a) Beam intensity map. (b) Isodose display for film measurement and DPM calculation; film is shown in solid and DPM in dashed lines. (c) One-dimensional profile comparisons between film measurement and DPM calculation; film is shown in solid and DPM in dashed lines. (d) Dose difference map in cGy: (DPM-film). (e) Dose difference map (in cGy) generated by applying a $1 \mathrm{~mm}$ gradient compensation. (f) Dose difference histogram of the dose difference map (dotted line) and the gradient compensated dose difference map (solid line).

the integration of this model with our in-house planning system and to commission the calculation method for clinical use, so that we can evaluate the clinical significance of using the accurate Monte Carlo dose calculation method for clinical IMRT planning.

\section{ACKNOWLEDGMENTS}

The authors would like to thank Dr. William R. Martin and Dr. Scott Wilderman for extremely useful discussions on the implementation of absolute dose and physics of DPM Monte Carlo code. We would also like to thank Bruce Curran and Sue Henshaw for their help with the film measurements. This work is supported by NIH Grant Nos. P01CA59872 and R01CA106770.

\footnotetext{
${ }^{a)}$ Author to whom correspondence should be addressed. Electronic mail: ntyagi@umich.edu

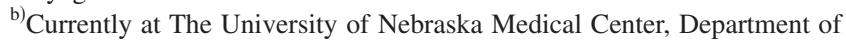
Radiation Oncology, Omaha, NE 68198-7521.

${ }^{1}$ G. A. Ezzell, J. M. Galvin, D. Low, J. R. Palta, I. Rosen, M. B. Sharpe, P. Xia, Y. Xiao, L. Xing, and C. X. Yu, "Guidance document on delivery, treatment planning, and clinical implementation of IMRT: Report of the IMRT Subcommittee of the AAPM Radiation Therapy Committee," Med. Phys. 30, 2089-2115 (2003).
} 
${ }^{2}$ F. C. du Plessis, C. A. Willemse, M. G. Lotter, and L. Goedhals, "Comparison of the Batho, ETAR and Monte Carlo dose calculation methods in CT based patient models," Med. Phys. 28, 582-589 (2001).

${ }^{3}$ W. U. Laub, A. Bakai, and F. Nusslin, "Intensity modulated irradiation of a thorax phantom: Comparisons between measurements, Monte Carlo calculations and pencil beam calculations," Phys. Med. Biol. 46, 1695-1706 (2001).

${ }^{4}$ D. W. O. Rogers, B. Walters, and I. Kawrakow, BEAMnrc Users Manual: NRC Report PIRS 509(a)revH. (2004).

5"MCNP-A general Monte Carlo N-Particle transport code," edited by J. F. Briesmeister (Report LA-12625-M, Los Alamos National Laboratory, Los Alamos NM, 1993).

${ }^{6}$ S. Agostinelli et al., "GEANT4-a simulation toolkit," Nucl. Instrum. Methods Phys. Res. A 506, 250-303 (2003).

${ }^{7}$ H. H. Liu, F. Verhaegen, and L. Dong, "A method of simulating dynamic multileaf collimators using Monte Carlo techniques for intensitymodulated radiation therapy," Phys. Med. Biol. 46, 2283-2298 (2001).

${ }^{8}$ E. Heath and J. Seuntjens, "Development and validation of a BEAMnrc component module for accurate Monte Carlo modeling of the Varian dynamic Millennium multileaf collimator," Phys. Med. Biol. 48, 40454063 (2003).

${ }^{9}$ J. O. Kim, J. V. Siebers, P. J. Keall, M. R. Arnfield, and R. Mohan, "A Monte Carlo study of radiation transport through multileaf collimators," Med. Phys. 28, 2497-2506 (2001).

${ }^{10}$ T. Pawlicki and C. M. Ma, "Monte Carlo simulation for MLC-based intensity-modulated radiotherapy," Med. Dosim. 26, 157-168 (2001).

${ }^{11}$ J. V. Siebers, P. J. Keall, J. O. Kim, and R. Mohan, "A method for photon beam Monte Carlo multileaf collimator particle transport," Phys. Med. Biol. 47, 3225-3249 (2002).

${ }^{12}$ R. F. Aaronson, J. J. DeMarco, I. J. Chetty, and T. D. Solberg, "A Monte Carlo based phase space model for quality assurance of intensity modulated radiotherapy incorporating leaf specific characteristics," Med. Phys. 29, 2952-2958 (2002).

${ }^{13}$ I. Chetty, J. J. DeMarco, and T. D. Solberg, "A virtual source model for Monte Carlo modeling of arbitrary intensity distributions," Med. Phys. 27, 166-172 (2000).

${ }^{14}$ J. Sempau, S. J. Wilderman, and A. F. Bielajew, "DPM, a fast, accurate Monte Carlo code optimized for photon and electron radiotherapy treatment planning dose calculations," Phys. Med. Biol. 45, 2263-2291
(2000).

${ }^{15}$ I. J. Chetty, P. M. Charland, N. Tyagi, D. L. McShan, B. A. Fraass, and A. F. Bielajew, "Photon beam relative dose validation of the DPM Monte Carlo code in lung-equivalent media," Med. Phys. 30, 563-573 (2003).

${ }^{16}$ I. Chetty, P. M. Charland, N. Tyagi, D. L. McShan, B. Fraass, and A. F. Bielajew, "Experimental validation of the DPM Monte Carlo code for photon beam dose calculations in inhomogeneous media," Med. Phys. 29, 1351 (2002).

${ }^{17}$ N. Tyagi, I. Chetty, B. Fraass, and A. Bielajew, "Calculations of a millennium multileaf collimator using the DPM and BEAM/DOSXYZ Monte Carlo codes," Med. Phys. 29, 1230-30 (2002).

${ }^{18}$ K. L. Lam, M. S. Muthuswamy, and R. K. Ten Haken, "Measurement of backscatter to the monitor chamber of medical accelerators using target charge," Med. Phys. 25, 334-338 (1998).

${ }^{19}$ A. F. Bielajew, Fundamentals of the Monte Carlo Method for Neutral and Charged Particle Transport (2001).

${ }^{20}$ M. N. Graves, A. V. Thompson, M. K. Martel, D. L. McShan, and B. A. Fraass, "Calibration and quality assurance for rounded leaf-end MLC systems," Med. Phys. 28, 2227-2233 (2001).

${ }^{21}$ T. R. Bortfeld, D. L. Kahler, T. J. Waldron, and A. L. Boyer, "X-ray field compensation with multileaf collimators," Int. J. Radiat. Oncol., Biol., Phys. 28, 723-730 (1994).

${ }^{22}$ D. W. Litzenberg, J. M. Moran, and B. A. Fraass, "Incorporation of realistic delivery limitations into dynamic MLC treatment delivery," Med. Phys. 29, 810-820 (2002).

${ }^{23}$ J. M. Moran, J. Radawski, and B. A. Fraass, "A dose gradient analysis tool for IMRT QA,” J. Appl. Clin. Med. Phys. 6, 62-73 (2005).

${ }^{24}$ B. Fraass, K. Doppke, M. Hunt, G. Kutcher, G. Starkschall, R. Stern, and J. Van Dyke, "American Association of Physicists in Medicine Radiation Therapy Committee Task Group 53: Quality assurance for clinical radiotherapy treatment planning," Med. Phys. 25, 1773-1829 (1998).

${ }^{25}$ P. M. Charland, I. J. Chetty, S. Yokoyama, and B. A. Fraass, "Dosimetric comparison of extended dose range film with ionization measurements in water and lung equivalent heterogeneous media exposed to megavoltage photons," J. Appl. Clin. Med. Phys. 4, 25-39 (2003).

${ }^{26}$ P. J. Muench, A. S. Meigooni, R. Nath, and W. L. McLaughlin, "Photon energy dependence of the sensitivity of radiochromic film and comparison with silver halide film and LiF TLDs used for brachytherapy dosimetry," Med. Phys. 18, 769-775 (1991). 